\title{
Integrating Genetic Data into Electronic Health Records: Medical Geneticists' Perspectives
}

\author{
Haleh Ayatollahi ${ }^{1,2}$, Seyedeh Fatemeh Hosseini ${ }^{2}$, Morteza Hemmat ${ }^{3,4}$ \\ ${ }^{1}$ Health Management and Economics Research Center, Iran University of Medical Sciences, Tehran, Iran \\ ${ }^{2}$ School of Health Management and Information Sciences, Iran University of Medical Sciences, Tehran, Iran \\ ${ }^{3}$ Social Determinants of Health Research Center, Saveh University of Medical Sciences, Saveh, Iran \\ ${ }^{4}$ Student Research Committee, Saveh University of Medical Sciences, Saveh, Iran
}

Objectives: Genetic disorders are the main causes of many other diseases. Integrating genetic data into Electronic Health Records (EHRs) can facilitate the management of genetic information and care of patients in clinical practices. The aim of this study was to identify the main requirements for integrating genetic data into the EHR system from the medical geneticists' perspectives. Methods: The research was completed in 2018 and consisted of two phases. In the first phase, the main requirements for integrating genetic data into the EHR system were identified by reviewing the literature. In the second phase, a 5-point Likert scale questionnaire was developed based on the literature review and the results derived from the first phase. Then, the Delphi method was applied to reach a consensus about the integration requirements. Results: The findings of the first phase showed that data elements, including patients' and healthcare providers' personal data, clinical and genetic data, technical infrastructure, security issues and functional requirements, should be taken into account before data integration. In the second phase, a consensus was reached for most of the items (mean $\geq 3.75$ ). The items with a mean value of less than 2.5 did not achieve a consensus and were removed from the final list. Conclusions: The integration of genetic data into the EHRs can provide a ground for increasing accuracy and precision in the diagnosis and treatment of genetic disorders. Such integration requires adequate investments to identify users' requirements as well as technical and non-technical issues.

Keywords: Genetics, Genetic Diseases, Medical Informatics, Electronic Health Records, User Requirements

Submitted: August 4, 2019

Revised: 1st, September 30, 2019; 2nd, October 28, 2019

Accepted: October 28, 2019

\section{Corresponding Author}

Morteza Hemmat

Social Determinants of Health Research Center, Saveh University of Medical Sciences, Saveh, Iran. Tel: +98-086-42343395, E-mail: mortezahemmat@gmail.com (https://orcid.org/0000-0003-3564-1791)

This is an Open Access article distributed under the terms of the Creative Commons Attribution Non-Commercial License (http://creativecommons.org/licenses/by$\mathrm{nc} / 4.0 /$ ) which permits unrestricted non-commercial use, distribution, and reproduction in any medium, provided the original work is properly cited.

(c) 2019 The Korean Society of Medical Informatics

\section{Introduction}

Genetic disorders include a wide range of illnesses. The main cause of genetic disorders is abnormalities in the structure of genes which, in turn, affects the normal functioning of human organs [1]. While the accessibility of information at the point of need can help to improve healthcare services, the collection and storage of genetic data are usually challenging tasks for genetic consultants and specialists. Hence, a number of genetic databases have been developed to improve the accessibility of information. Such a database can help to diagnose and follow up genetic disorders [2,3]. Because genetic data are part of a patient's medical history, the integration of these data into Electronic Health Records (EHRs) has been suggested to facilitate clinical activities in the absence 
of genetic specialists and to accelerate the process of clinical decision making [3].

The integration of genetic data into the EHRs can be useful for clinical practices, teaching clinical genetics, conducting genetic research, and saving time and cost. However, given the complexity of genetic data and the differences between these data and other clinical data, the process of integration seems difficult $[4,5]$. Such integration also requires compliance with existing standards, such as ISO/TS 20428 [6], Health Level 7, Fast Healthcare Interoperability Resources (HL7 FHIR) genomics implementation guidance [7] and the Global Alliance for Genomics and Health (GA4GH) documents [8].

According to the literature, the integration of genetic data into EHRs has received more attention in the last decade. Hall et al. [9] discussed current challenges in and opportunities for merging EHRs data and genomics for cardiovascular studies. They suggested that the ideal situation can be storing genomic data along with EHR data. Moreover, a number of review studies have been published to show the capacity of EHRs and the benefits of linking genomic data to other patient data $[10,11]$ as well as ethical, logistic, and technological issues involved in incorporating these data into EHRs [12]. In another study, Stark et al. [13] reviewed the diversity of approaches and recent progress made by national genomic-medicine initiatives in the United Kingdom, France, Australia, and the United States and provided a roadmap for sharing strategies, standards, and data internationally. They also suggested that the implementation of genomic medicine would have benefits for individual patients, families, and healthcare systems. To achieve these benefits, a number of projects have been conducted around the world. In the US, eMERGE (Electronic Medical Records and genomics) project was started in 2007 to integrate genomic data into EHRs and to use genetic test results in clinical and pharmaceutical research $[14,15]$. Integrating cancer genomic data into patient EHRs was another initiative started in the US in 2014 [16]. In the UK, it was announced that a fully integrated Genomic Medicine Service (GMS) would be established in October 2018 [17]. The Australian government developed the National Health Genomics Policy Framework (NHGPF) in recognition of the need for using genomic technology across the health system. The framework was endorsed in November 2017 by the Council of Australian Governments (COAG) Health Council, and it represents a shared commitment to the implementation of genomic technology into health systems for the benefit of all Australians [18]. In Iran, however, the recording and storage of genetic data are performed ei- ther manually or electronically, and they are not integrated into the rest of the patient's medical records or the health system. As the country has begun to develop the necessary infrastructure for implementing EHRs, and there is a need for better communication between the current and future systems, this study aimed to identify the main requirements for integrating genetic data into the EHR system from the medical geneticists' perspectives. It is expected that the availability of data improves clinical practices, patient outcomes, and research activities.

\section{Methods}

This was a mixed methods study, which was completed in 2018. Before the research was conducted, ethics approval was obtained from the Institutional Review Board of Iran University of Medical Sciences (No. IR.IUMS. REC.1394.9211304204). This study consisted of two phases. In the first phase, the main requirements for integrating genetic data into EHRs were identified via literature review. To achieve this, three countries, namely, the US, the UK, and Australia, which had more experience in the field of study, were selected. Then, papers and published documents related to the integration of genetic data into patient care records or into EHRs were searched via various databases, such as PubMed, Scopus, Web of Science, and Google Scholar. To search the papers and documents, keywords, such as genetic data, EHRs, patient care records, integration, and the name of each country were used. As genetic data have to be used along with the data stored in the EHRs, the documents related to the EHRs of the selected countries were also searched and reviewed. The reviewed documents included 'Electronic Medical Records and Genomics (eMERGE) Network' [15], 'Genomics and Genome Editing in the NHS' [17], 'Annual report of the Chief Medical Officer 2016, generation genome' [19] 'National Health Genomics Policy Framework 2018-2021' [20], and papers related to the topic of the study [9-14,16,18,21-27]. In the second phase, a questionnaire was designed based on the results derived from the first phase of the study and the literature review. Then, the experts in the field of medical genetics were asked to complete the questionnaire and determine the integration requirements in Iran.

\section{Research Participants}

In the second phase, the Delphi method was applied in three rounds. In the first round, the participants $(n=30)$ included 27 faculty members in the field of medical genetics who 
worked in medical universities across the country and three genetic counselors who worked in the Ministry of Health. In the second round, 20 faculty members and one genetic counselor and in the third round, 13 faculty members took part in the study. In each round of the Delphi study, the participants of the previous round were invited to take part in the next round.

\section{Research Instruments}

In the first phase, a form was designed to extract the main requirements for integrating genetic data into EHRs. As a result, a list of items including data elements (patient's data, healthcare provider's data, clinical and genetic data), the main requirements for technical infrastructure, security issues, and the main functional requirements was developed based on the literature review [9-27]. In the second phase, the results of the first phase were used to develop a 5-point Likert scale questionnaire with scores ranging from 'very important' (5) to 'unimportant' (1). The questionnaire consisted of four sections-data elements (patient's 11 items, healthcare provider's 10 items, clinical 19 items, and genetic data 13 items), technical infrastructure (6 items), and security (6 items), and functional requirements ( 16 items). In the second and the third round of the Delphi study, a new questionnaire with 23 items (2nd round) and 7 items (3rd round) was designed and distributed to the participants. The questionnaire items where those for which no consensus was achieved in the previous round.

\section{Data Analysis}

In the first phase, the method of content analysis was used to extract data from the literature. Each paper was screened carefully to identify the data elements and functional requirements for integrating genetic data into EHRs. In the second phase, descriptive statistics (e.g., mean value and standard deviation) were used to analyze the data.

As a 5-point Likert scale questionnaire was used to collect data, and its scores ranged from 1 to 5 , two cutoff points were considered to show reaching or not reaching a consensus. If more than $75 \%$ of the respondents agreed with an item (mean 23.75 ), it meant that a consensus had been reached, and if $50 \%$ to $75 \%$ of the respondents agreed with an item, the item would be asked again in the second round of the Delphi study. Items with a level of agreement less than $50 \%$ (mean $\leq 2.5$ ) would be removed from the final list, and this showed that a consensus had not been reached [28].

\section{Results}

In the first phase of the study, a number of papers and documents in which the current state of integrating genetic data into EHRs were reported in the US, the UK, and Australia were reviewed carefully, and the main requirements for integrating genetic data into EHRs were extracted. The results of the first phase of this study showed that the main requirements for integrating genetic data into EHRs could be categorized in four areas: data elements (patients', healthcare

Table 1. Functional requirements for integrating genetic data into the EHRs in the selected countries

\begin{tabular}{lccc}
\multicolumn{1}{c}{ Functional requirements } & USA & UK & Australia \\
\hline Drawing a genetic pedigree & $\sqrt{ }$ & $\sqrt{ }$ & $\mathrm{x}$ \\
Genetic risk assessment & $\sqrt{ }$ & $\sqrt{ }$ & $\sqrt{ }$ \\
Availability of a patient portal & $\sqrt{ }$ & $\sqrt{ }$ & $\sqrt{ }$ \\
Availability of genetic data for research & $\sqrt{ }$ & $\sqrt{ }$ & $\sqrt{ }$ \\
Availability of a patient's health records summary & $\sqrt{ }$ & $\sqrt{ }$ \\
Information sharing between genetic information systems and other systems, & & $\sqrt{ }$ \\
such as laboratory information systems & $\sqrt{ }$ & $\sqrt{ }$ \\
Presenting gene sequences & $\sqrt{ }$ & $\sqrt{ }$ \\
Genomics data analysis & $\sqrt{ }$ & $\sqrt{ }$ \\
Statistical reporting & $\sqrt{ }$ & $\sqrt{ }$ \\
Updating genetic related data in the EHRs & $\sqrt{ }$ & $\sqrt{ }$ \\
Searching genetic related data in the EHRs & $\mathrm{x}$ & $\sqrt{ }$ \\
Reporting missing genetic data & $\sqrt{ }$ \\
Retrieving unstructured genetic data & $\mathrm{x}$ & $\mathrm{x}$
\end{tabular}

EHR: Electronic Health Records. 
providers', clinical, and genetic data), technical infrastructure, security and functional requirements. In the US and the UK, a wide range of data elements were collected, whereas in Australia, some data elements, such as nationality, diet, smoking, physical examinations, vaccination history, and gene therapy history were not collected. The technical infrastructures used for the systems were similar in all selected countries; however, the security issues were considered differently. For example, encryption at the time of sending and receiving information and storing data in EHRs was not considered in the UK and Australia. In terms of the functional requirements, the systems used in the US appeared to be more functional (Table 1). For example, the possibility of genetic data analysis was not considered in the systems used in the UK and Australia.

In the second phase, the Delphi method was used and medical geneticists were asked to identify the main requirements for integrating genetic data into EHRs. All participants had a $\mathrm{PhD}$ degree in medical genetics. The participants' demographic information in three rounds of the Delphi study is presented in Table 2.

The findings of the Delphi study showed that from the medical geneticists' perspectives, most of the data elements mentioned in the questionnaire were important for integrating genetic data into EHRs and a consensus was reached for them. Among the patient data, the highest mean values were related to the national ID (4.73 \pm 0.64$)$, patient's name (4.53 $\pm 0.57)$ and surname $(4.53 \pm 0.57)$. Among the clinical data, the highest mean values belonged to cancer history (4.93 \pm $0.25)$, family history of diseases $(4.60 \pm 0.85)$, and the chief complaint $(4.60 \pm 0.50)$. Regarding the genetic data elements, while all of the items mentioned in the questionnaire were found to be important by the respondents, the highest mean values were related to the interpretation of genetic test results $(4.83 \pm 0.38)$, a genetic pedigree $(4.60 \pm 0.50)$, and the genetic test results $(4.57 \pm 0.57)$. Regarding the healthcare provider's data, most of the data elements were found to be important; however, the highest mean values belonged to medical geneticist's name, surname, and ID $(4.10 \pm 0.30)$.

Finally, from the list of data elements, those items with a mean value between 2.5 and 3.75 were selected to be asked again in the next round of the Delphi study to reach a consensus. Some of these items were date of death $(3.40 \pm 0.81)$; patient's medical record number ( $3.57 \pm 0.97)$; blood group ( $3.33 \pm 0.96)$; weight $(3.03 \pm 0.89)$; vaccination history (2.87 $\pm 0.82)$; the name of the healthcare center $(3.10 \pm 1.18)$; and referring physician's name, surname, and ID (3.30 \pm 1.02$)$.

All of the technical requirements mentioned in the questionnaire were found to be important by the respondents. In this section, the highest mean values belonged to the usable interface design ( $4.77 \pm 0.43)$ and the use of standard thesaurus terms (4.73 \pm 0.45$)$. Regarding security issues, as Table 3 shows, consensus was reached for all of the mentioned items, and the highest mean values were related to the necessity of encryption at the time of sending and receiving information (4.77 \pm 0.43$)$, and the necessity of developing confidentiality guidelines for information sharing $(4.77 \pm 0.43)$.

Regarding the functional requirements, most of the system functions mentioned in the questionnaire were found to be important by the participants, and a consensus was reached for them. In this section, the highest mean values were related to the possibility of drawing a genetic pedigree (4.77 \pm 0.43 ) and searching genetic-related data in EHRs (4.67 \pm $0.55)$. Those items for which a consensus was not reached

Table 2. Participants' demographic information in three rounds of the Delphi study

\begin{tabular}{|c|c|c|c|c|c|c|c|c|c|}
\hline \multirow{3}{*}{ Variable } & \multicolumn{9}{|c|}{ Workplace } \\
\hline & \multicolumn{3}{|c|}{ Ministry of Health } & \multicolumn{3}{|c|}{ Medical universities } & \multicolumn{3}{|c|}{ Total } \\
\hline & 1 st round & 2 nd round & 3 rd round & 1 st round & 2 nd round & 3 rd round & 1 st round & 2nd round & 3rd round \\
\hline \multicolumn{10}{|l|}{ Age (yr) } \\
\hline $30-34$ & $1(3.3)$ & 0 & 0 & $1(3.3)$ & $1(4.8)$ & 0 & $2(6.6)$ & $1(4.8)$ & 0 \\
\hline $35-39$ & $1(3.3)$ & 0 & 0 & $4(13.4)$ & $5(23.8)$ & $2(15.4)$ & $5(16.7)$ & $5(23.8)$ & $2(15.4)$ \\
\hline $40-44$ & 0 & 0 & 0 & $5(16.7)$ & $4(19)$ & $3(23.1)$ & $5(16.7)$ & $4(19)$ & $3(23.1)$ \\
\hline $45-49$ & 0 & 0 & 0 & $8(26.7)$ & $5(23.8)$ & $3(23.1)$ & $8(26.7)$ & $5(23.8)$ & $3(23.1)$ \\
\hline$\geq 50$ & $1(3.3)$ & $1(4.8)$ & 0 & $9(30)$ & $5(23.8)$ & $5(38.4)$ & $10(33.3)$ & $6(28.6)$ & $5(38.4)$ \\
\hline \multicolumn{10}{|l|}{ Sex } \\
\hline Male & 0 & 0 & 0 & $20(66.7)$ & $13(61.9)$ & $10(77)$ & $20(66.7)$ & $13(61.9)$ & $10(77)$ \\
\hline Female & $3(10)$ & $1(4.8)$ & 0 & $7(23.3)$ & $7(33.3)$ & $3(23)$ & $10(33.3)$ & $8(38.1)$ & $3(23)$ \\
\hline
\end{tabular}

Values are presented as frequency (\%). 
Table 3. Security requirements for integrating genetic data into the EHRs

\begin{tabular}{|c|c|c|c|c|c|c|c|c|c|c|}
\hline \multirow{2}{*}{$\begin{array}{l}\text { Security } \\
\text { requirements }\end{array}$} & \multicolumn{5}{|c|}{ Degree of importance } & \multirow{2}{*}{$\begin{array}{l}\text { Median / } \\
\text { IQR }\end{array}$} & \multirow{2}{*}{$\begin{array}{c}\text { First } \\
\text { quartile }\end{array}$} & \multirow{2}{*}{$\begin{array}{c}\text { Third } \\
\text { quartile }\end{array}$} & \multirow[b]{2}{*}{ Mean \pm SD } & \multirow[b]{2}{*}{ Consensus } \\
\hline & $\begin{array}{c}\text { Very } \\
\text { important }\end{array}$ & Important & Neutral & $\begin{array}{l}\text { Slightly } \\
\text { important }\end{array}$ & Unimportant & & & & & \\
\hline $\begin{array}{l}\text { Obtaining } \\
\text { informed } \\
\text { consent } \\
\text { from patients }\end{array}$ & $19(63.3)$ & $11(36.7)$ & 0 & 0 & 0 & $5 / 1$ & 4 & 5 & $4.63 \pm 0.49$ & $\sqrt{ }$ \\
\hline $\begin{array}{l}\text { Encryption at the } \\
\text { time of sending } \\
\text { and receiving } \\
\text { information }\end{array}$ & 23 (67.7) & $7(23.3)$ & 0 & 0 & 0 & $5 / 0.25$ & 4.75 & 5 & $4.77 \pm 0.43$ & $\sqrt{ }$ \\
\hline $\begin{array}{l}\text { Encryption at the } \\
\text { time of storing } \\
\text { data } \\
\text { in the EHRs }\end{array}$ & $18(60.0)$ & $12(40.0)$ & 0 & 0 & 0 & $5 / 1$ & 4 & 5 & $4.60 \pm 0.50$ & $\sqrt{ }$ \\
\hline $\begin{array}{l}\text { Using a separate } \\
\text { password for } \\
\text { each user of } \\
\text { the EHRs }\end{array}$ & $15(50.0)$ & $14(46.7)$ & $1(3.3)$ & 0 & 0 & $4.5 / 1$ & 4 & 5 & $4.47 \pm 0.57$ & $\sqrt{ }$ \\
\hline $\begin{array}{l}\text { User } \\
\text { authorization } \\
\text { to get access to } \\
\text { the genetic data }\end{array}$ & $12(40.0)$ & $17(56.7)$ & $1(3.3)$ & 0 & 0 & $4 / 1$ & 4 & 5 & $4.37 \pm 0.57$ & $\sqrt{ }$ \\
\hline $\begin{array}{l}\text { Developing } \\
\text { confidentiality } \\
\text { guidelines for } \\
\text { information } \\
\text { sharing }\end{array}$ & $23(76.7)$ & $7(23.3)$ & 0 & 0 & 0 & $5 / 0.25$ & 4.75 & 5 & $4.77 \pm 0.43$ & $\sqrt{ }$ \\
\hline
\end{tabular}

Values are presented as frequency (\%).

EHR: Electronic Health Records, IQR: interquartile range.

were asked again in the second round of the Delphi study. Some of these items were the availability of a patient portal $(3.40 \pm 0.62)$, presenting gene sequences $(3.57 \pm 1.50)$, genomics data analysis $(3.27 \pm 1.41)$, and reporting missing genetic data $(3.70 \pm 0.84)$.

In the second and the third round of the Delphi study, the data elements and the functional requirements for which a consensus had not been reached in the previous round were asked again. Finally, those items with a mean value less than 2.5 for which no consensus was reached were removed from the final list of the main requirements for integrating genetic data into EHRs. Among the data elements, diet (2.19 $\pm 0.51)$, smoking $(2.19 \pm 0.51)$, alcohol use $(2.28 \pm 0.64)$, the name of the healthcare center $(2.31 \pm 0.85)$, and among the functional requirements retrieving unstructured genetic data
$(2.38 \pm 1.32)$ were removed, and the rest of the items reached a consensus.

\section{Discussion}

The importance of integrating genetic data into the EHRs has been considered in several studies $[5,9,10]$. UllmanCullere and Mathew [5] stated that structured genetic test results can be transmitted from the laboratory into EHRs with sufficient data structure for clinical decision support; however, much work remains to establish mature clinical genomic standards for broad adoption into EHRs. Hall et al. [9] noted that the interoperability of EHRs could allow genetic test results to be available at points of care, including dispensing pharmacies and outpatient clinics. Glicksberg et al. 
[10] believed that EHR-linked biobanks provide an excellent opportunity for unconventional analyses, which can result in surprising findings.

In the current research, the main requirements for integrating genetic data into EHRs were identified to improve clinical practices. These requirements were categorized in four areas: data elements, technical infrastructure, security, and functional requirements. Regarding data elements, patient's and healthcare provider's personal data as well as clinical and genetic data were found to be important. Similarly, Warner et al. [16] emphasized that the integration of clinical data with genetic data in EHRs is important. In another study, Robinson et al. [11] indicated that phenotypic clinical documentation along with laboratory data, medication receipts, family history, and environmental exposures makes the EHR a practical data source to be used in genomic studies. It is notable that, although there are documents in which data elements and necessary metadata have been defined to be used in a structured clinical genomic sequencing report and in the EHRs [6-8], few studies have addressed the required data elements and system functions from users' perspectives in clinical practices.

Regarding the technical infrastructure, the results of the first and the second phase of the research showed that using unified modeling language (UML), the standard thesaurus terms, a standard format for information sharing with laboratory and pharmacy information systems, a secure network, and a usable interface were important. Similarly, Shoenbill et al. [12] discussed a number of technical issues related to the integration of genetic data into EHRs. They believed that system developers must agree on standardized genetic terminology and methods of data exchange. As there is a large volume of data on the human genome and its interpretation, genomic data storage must be decreased and new innovations in data storage and computation, such as cloud-based computing need to be taken into account.

The security requirements identified in this study included obtaining informed consent from the patient, encryption at the time of sending and receiving data, encryption at the time of data storage, using a separate password for each user of EHRs, user authorization to obtain access to the genetic data, and developing confidentiality guidelines for information sharing. The findings are in line with the results of other similar studies in which data encryption and user authorization have been discussed as security solutions for the integration of genetic data into the EHRs [3,29]. Apart from the mentioned items, Mehraeen et al. [30] showed that information security in health information systems should be respected in terms of administrative, technical, and physical safeguards, and relevant solutions should be formulated for each area. Shoenbill et al. [12] indicated that the risk of confidentiality breach can be minimized by ensuring high security in the use, storage, and sharing of genetic data. It is also essential to ensure that the de-identification and security of data are at a high level, particularly as various cloud-based approaches might be used to manage big data [9].

In terms of the functional requirements, the findings showed that a number of functions, such as the possibility of drawing a genetic pedigree, genetic risk assessment, availability of a patient portal, and information sharing between genetic information systems and other health information systems were important from the perspective of medical geneticists. Similarly, Scheuner et al. [3] found that the mentioned functions were important and the interpretation of genetic test results can be added to the Clinical Decision Support Systems to facilitate decision-making in clinical practices. Shirts et al. [4] indicated that developing interoperable systems to receive and display genetic and/or genomic data along with alerting clinicians is essential for clinical care. Overall, the results showed that genetic information and genetic test results should be available in EHRs, and due to the complexity of this type of data, extra effort is needed to address the users' requirements in terms of technical, security, and functional issues.

The results can be useful in developing new integrated systems, which, in turn, can support clinical practices and research purposes. Such a system can help clinicians in making clinical decisions and can encourage them to use data in personalized medicine and in their routine care plans. As the requirements might be different in each country, and due to the complexity of genetic data and the challenges of integrating these data into EHRs, more theoretical and practical studies are needed to provide a ground for implementing a successful integrated system.

In this study, the requirements for integrating genetic data into EHRs were identified. However, this research had some limitations. Firstly, only three countries were selected and included in the first phase to identify the main requirements for integration. Although the number of countries was limited, their experience regarding the integration of genetic data into the EHRs was valuable, and they were among the first countries that have started such projects. Secondly, in this research, the findings were derived from reviewing the literature and applying the Delphi method. It seems that conducting a qualitative study could help to gain a better understanding of users' requirements. Thirdly, the main 
purpose of this manuscript was to identify data elements and system functions required for clinical practices. While the availability of genetic data can facilitate research projects, genomic researchers may need many specific types of data elements for genomic research activities that may not be included in the EHR, and other databases should be able to support the researchers' requirements. Therefore, users' requirements can vary depending on their purposes and need to be investigated in detail.

\section{Conflict of Interest}

No potential conflict of interest relevant to this article was reported.

\section{Acknowledgments}

This study was funded and supported by Iran University of Medical Sciences (No. IUMS/SHMIS_1394/9211304204).

\section{ORCID}

Haleh Ayatollahi (http://orcid.org/0000-0003-3974-3648)

Seyedeh Fatemeh Hosseini (http://orcid.org/0000-0001-5548-9737)

Morteza Hemmat (http://orcid.org/0000-0003-3564-1791)

\section{References}

1. Shiva N, Tachi SK, Fatemeh AG. Survey of parents' knowledge regarding their children's genetic diseases. Genet Third Millenn 2011;9(1):2285-9.

2. Talebi V. Comparative study of genetic information management system in selected the countries and presentation a model for Iran [dissertation]. Tehran, Iran: Tehran University of Medical Sciences; 2012.

3. Scheuner MT, de Vries H, Kim B, Meili RC, Olmstead $\mathrm{SH}$, Teleki S. Are electronic health records ready for genomic medicine? Genet Med 2009;11(7):510-7.

4. Shirts BH, Salama JS, Aronson SJ, Chung WK, Gray SW, Hindorff LA, et al. CSER and eMERGE: current and potential state of the display of genetic information in the electronic health record. J Am Med Inform Assoc 2015;22(6):1231-42.

5. Ullman-Cullere MH, Mathew JP. Emerging landscape of genomics in the Electronic Health Record for personalized medicine. Hum Mutat 2011;32(5):512-6.

6. International Organization for Standardization. Health informatics: data elements and their metadata for de- scribing structured clinical genomic sequence information in electronic health records. Geneva, Switzerland: International Organization for Standardization; 2017. (ISO/TS 20428:2017).

7. Health Level Seven International. Genomic implementation guidance [Internet]. Ann Arbor (MI): Health Level Seven International; 2018 [cited at 2019 Oct 25]. Available from: https://www.hl7.org/fhir/genomics. html.

8. Van Baren J. GA4GH Schemas Documentation Release 0.0.1 [Internet]. Toronto, Canada: Global Alliance for Genomics \& Health; 2018 [cited at 2019 Oct 25]. Available from: https://buildmedia.readthedocs.org/media/ pdf/ga4gh-schemas/latest/ga4gh-schemas.pdf.

9. Hall JL, Ryan JJ, Bray BE, Brown C, Lanfear D, Newby LK, et al. Merging electronic health record data and genomics for cardiovascular research: a science advisory from the American Heart Association. Circ Cardiovasc Genet 2016;9(2):193-202.

10. Glicksberg BS, Johnson KW, Dudley JT. The next generation of precisionmedicine: observational studies, electronic health records, biobanks and continuous monitoring. Hum Mol Genet 2018;27(R1):R56-R62.

11. Robinson JR, Wei WQ, Roden DM, Denny JC. Defining phenotypes from clinical data to drive genomic research. Annu Rev Biomed Data Sci 2018;1:69-92.

12. Shoenbill K, Fost N, Tachinardi U, Mendonca EA. Genetic data and electronic health records: a discussion of ethical, logistical and technological considerations. J Am Med Inform Assoc 2014;21(1):171-80.

13. Stark Z, Dolman L, Manolio TA, Ozenberger B, Hill SL, Caulfied MJ, et al. Integrating genomics into healthcare: a global responsibility. Am J Hum Genet 2019;104(1):13-20.

14. Gottesman O, Kuivaniemi H, Tromp G, Faucett WA, Li R, Manolio TA, et al. The Electronic Medical Records and Genomics (eMERGE) network: past, present, and future. Genet Med 2013;15(10):761-71.

15. Center for Genetic Medicine. Electronic Medical Records and Genomics (eMERGE) Network [Internet]. Chicago (IL): Northeastern University; 2018 [cited at 2019 Oct 25]. Available from: https://www.cgm.northwestern.edu/research/emerge-network/index.html.

16. Warner JL, Jain SK, Levy MA. Integrating cancer genomic data into electronic health records. Genome Med 2016;8(1):113.

17. House of Commons of the United Kingdom. Genomics and genome editing in the NHS [Internet]. London, UK: 
House of Commons of the United Kingdom; 2018 [cited at 2019 Oct 25]. Available from: https://publications.parliament.uk/pa/cm201719/cmselect/cmsctech/349/349. pdf.

18. Burns BL, Bilkey GA, Coles EP, Bowman FL, Beilby JP, Pachter NS, et al. Healthcare system priorities for successful integration of genomics: an Australian focus. Front Public Health 2019;7:41.

19. Davies SC. Annual report of the Chief Medical Officer 2016; generation genome [Internet]. London, UK: Department of Health; 2017 [cited at 2019 Oct 25]. Available from: https://assets.publishing.service.gov.uk/ government/uploads/system/uploads/attachment_data/ file/631043/CMO_annual_report_generation_genome. pdf.

20. The Department of Health. National Health Genomics Policy Framework 2018-2021 [Internet]. Canberra, Australia: The Department of Health; 2017 [cited at 2019 Oct 25]. Available from: http://www.health.gov.au/ internet/main/publishing.nsf/Content/national-healthgenomics-policy-framework-2018-2021.

21. O'Daniel JM, McLaughlin HM, Amendola LM, Bale SJ, Berg JS, Bick D, et al. A survey of current practices for genomic sequencing test interpretation and reporting processes in US laboratories. Genet Med 2017;19(5):575-82.

22. Rasmussen LV, Overby CL, Connolly J, Chute CG, Denny JC, Freimuth R, et al. Practical considerations for implementing genomic information resources. Experiences from eMERGE and CSER. Appl Clin Inform 2016;7(3):870-82.

23. Orlando LA, Sperber NR, Voils C, Nichols M, Myers
RA, Wu RR, et al. Developing a common framework for evaluating the implementation of genomic medicine interventions in clinical care: the IGNITE Network's Common Measures Working Group. Genet Med 2018;20(6):655-63.

24. Sperber NR, Carpenter JS, Cavallari LH, J Damschroder L, Cooper-DeHoff RM, Denny JC, et al. Challenges and strategies for implementing genomic services in diverse settings: experiences from the Implementing GeNomics In pracTicE (IGNITE) network. BMC Med Genomics 2017;10(1):35.

25. The 100000 Genomes Project: bringing whole genome sequencing to the NHS. BMJ 2018;361:k1952.

26. Global Alliance for Genomics and Health. GENOMICS: a federated ecosystem for sharing genomic, clinical data. Science 2016;352(6291):1278-80.

27. Gaff CL, M Winship I, M Forrest S, P Hansen D, Clark J, M Waring P, et al. Preparing for genomic medicine: a real world demonstration of health system change. NPJ Genom Med 2017;2:16.

28. Gerrish K, Lacey A. The research process in nursing. Chichester, UK: Wiley-Blackwell; 2010.

29. Health Level Seven International. HL7 Domain Analysis Model: Clinical Genomics, Release1 [Internet]. Ann Arbor (MI): Health Level Seven International; 2018 [cited at 2019 Oct 25]. Available from: http://www.hl7. org/implement/standards/product_brief.cfm?product_ $\mathrm{id}=479$.

30. Mehraeen E, Ayatollahi H, Ahmadi M. Health information security in hospitals: the application of security safeguards. Acta Inform Med 2016;24(1):47-50. 